\title{
Protective Effects of Silymarin and/or Omega-3 Fatty Acids against Paracetamol-initiated Liver Toxicity in Rats
}

\author{
Rajesh A. Maheshwari ${ }^{1 *}$, Divya Khemani', Ashim Kumar Sen1, Ramachandran Balaraman', \\ Ramaswamy Velmurugan², Dhanya B. Sen ${ }^{1}$ \\ 1'Department of Pharmacy, Sumandeep Vidyapeeth (Deemed to be University), Piparia, Waghodia, Vadodara, Gujarat, INDIA. \\ 2Drug Discovery R\&D, Century Pharmaceutical Limited, Vadodara, Gujarat, INDIA.
}

\begin{abstract}
Background and Aim: The proposed experiment was planned to evaluate the potential protective function of aqueous extract of Silymarin (SL) concentrate and/or Omega-3 fatty acids (O3FAs) against PCM-initiated liver toxicity in rats. Materials and Methods: Thirty Wistar-albino rats (either of sex) were distributed among five equivalent groups as follows: Normal control group, PCM control group, PCM + SL group $125 \mathrm{mg} / \mathrm{kg}$, p.o.), PCM + O3FAs group (300 mg/kg, p.o.), PCM + SL + O3FAs group. All the treatments were administered for 14 days. Hepatotoxicity was induced in all groups except Group I by injection of PCM $\left(2 \mathrm{~g} / \mathrm{kg}\right.$, i.p.) on $14^{\text {th }}$ day post the administration of drugs. Results: Noticeable rise in the levels of AST, ALT, ALP, total bilirubin, direct bilirubin, MDA, TNF- $\alpha$, IL-6, VEGF and CRP were seen alongside a substantial reduction in the levels of serum total protein content and antioxidant enzymes, SOD, GSH, catalase in PCM control group. Treatment with SL or O3FAs or SL + O3FAs exhibited a considerable alteration in abovementioned biomarkers. Conclusion: The study concluded that SL and/or O3FAs protected the liver against PCM-initiated liver toxicity. However, the significantly increased hepatoprotective effects of SL when combined with O3FAs insinuate a possible synergism.
\end{abstract}

Key words: Silymarin, Omega-3 fatty acids, TNF- $\alpha$, IL-6, VEGF, CRP.

\section{INTRODUCTION}

Paracetamol (PCM) is a safe and efficient medication used to treat pain and fever when consumed at therapeutic levels. ${ }^{1}$ Besides its effectiveness, acute overdose of PCM is known to produce a fatal adverse effect namely centrilobular hepatic necrosis. ${ }^{2}$

Several study reports were stated the involvement of reactive oxygen species (ROS) in the pathway of liver toxicity induced by PCM. Superoxide, free radicals and nitric oxide tend to serve as chief mediators of liver tissue injuries. ${ }^{3,4}$ Presently, PCM-initiated liver damage is a common mechanistic model for evaluating herbal remedies and other hepatoprotective interventions.
Liver toxicity initiated by PCM has a pathophysiology coupled with the production of free radicals and reactive oxygen metabolites. It has been declared that among the primary perspectives adopted to improve or develop a protection for PCM-initiated liver toxicity, the ultimate rational efficacy has been seen by the use of antioxidant agents. Antioxidants possess intense scavenging activity towards free radicals in order to eradicate toxicities induced by PCM.

Liver is the vital organ responsible for most of the biological actions in human body. Detoxification of toxic substances is the foremost function of liver. ${ }^{5}$ Managing liver ailments is a challenging task for the medical fraternity even today. Hence, investigation
Submission Date: 01-12-2020; Revision Date: 10-02-2021; Accepted Date: 03-05-2021.

DOI: 10.5530/ijper.56.1s.46 Correspondence: Dr. Rajesh A Maheshwari Department of Pharmacy, Sumandeep Vidyapeeth (Deemed to be University), Piparia, Waghodia, Vadodara-391760, Gujarat, INDIA.

Email id: rajpharma2007@gmail. com

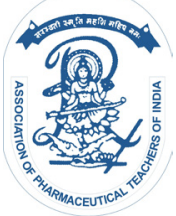

www.ijper.org 
on more effectual and harmless hepatoprotective medications has continued to be a crucial research area. Since long, herbal remedies are utilized for the management of liver ailments. Synthetic and traditional medicines presently available are not effective for curing liver ailments. Therefore, obtaining novel medicines from herbal sources with minimal side effects is becoming really essential these days. ${ }^{6}$

Silymarin (SL) contain polyphenolic constituent extracted from the fruits and seeds of Silybum marianum (Asteraceae family). ${ }^{7}$ Omega-3 fatty acids (O3FAs) serve to be essential fatty acids and are provided with amplified health benefits. The hepatoprotective activity of SL or O3FAs in PCM intoxication have been earlier depicted. ${ }^{8,9}$

The proposed work was planned to assess the possible protective role of aqueous extract of (SL) and/or Omega-3 fatty acids (O3FAs) against PCM-initiated liver toxicity in rats.

\section{MATERIALS AND METHODS}

\section{Drugs and chemicals}

Paracetamol formulation marketed by Zydus Cadila, Ahmedabad, India was procured from authorized retailer. Silymarin and O3FAs were obtained from Alpsure Life Sciences Pvt. Ltd., New Delhi. Alkaline phosphatase (ALP), Alanine transaminase (ALT), Aspartate transaminase (AST), bilirubin, total protein kits utilized in the protocol were procured from ARKRAY Healthcare Pvt. Ltd., Surat, India and Transasia Bio-Medicals Limited, India. Interleukin 6 (IL-6), Tumor Necrosis Factor-alpha (TNF- $\alpha$ ), Vascular endothelial growth factor (VEGF), C - reactive protein (CRP) ELISA kits were purchased from Sigma Aldrich. Remaining substances utilized in the protocol were of analytical grade.

\section{Experimental animals}

This research work was performed on healthy fullygrown albino Wistar rats of either sex weighing 150-200 gm. Rats were kept under optimal environment (Light/ dark cycle: $12 \mathrm{~h}$, Temperature: $24^{\circ} \mathrm{C}$, Humidity: 35 to $60 \%$ ), contained in polypropylene cages having free access to optimum quality of diet and drinking water ad libitum. Prior approval from Institutional Animal Ethics Committee (IAEC) was taken for experiment on animals.

\section{Experimental design}

Animals were distributed equally among 5 different groups each comprising of 6 animals. Group I labeled as normal control group and was given $10 \mathrm{ml} / \mathrm{kg} /$ day of normal saline. Group II labeled as PCM control group and were given $10 \mathrm{ml} / \mathrm{kg} /$ day of normal saline. Group III animals received aqueous extract of SL (25 mg/kg, p.o.), ${ }^{10}$ Group IV animals received O3FAs (300 mg/kg, p.o.), ${ }^{9}$ Group V received combination of aqueous extract of SL + O3FAs. All the aforementioned test compounds were administered by gavage method consecutively for 14 days wherein animals were fasted for 3-4 hrs. prior to and $1 \mathrm{hr}$. after administration to ensure proper absorption. Hepatotoxicity was induced in all groups expect Group I by injection of PCM (2 g/ $\mathrm{kg}$, i.p.) on $14^{\text {th }}$ day post administration of drugs. ${ }^{10}$ Rats were sacrificed after 48 hrs of PCM administration under mild anesthesia.

On completion of test duration, blood samples were collected (2-3 ml) from anesthetized animals via retroorbital plexus puncture using glass capillaries. The collected blood samples, after a standing time of $1 / 2 \mathrm{hr}$ were centrifuged in Remi Centrifuge at the speed of 2500 $\mathrm{rpm}(10 \mathrm{~min})$. The separated serum was stored at $-20^{\circ} \mathrm{C}$ for evaluation of liver functions tests viz. AST, ALT, ALP, total bilirubin, total protein and direct bilirubin from serum using standard diagnostic kit (ARKRAY Healthcare Pvt. Ltd., Surat, India and Transasia BioMedicals Limited, India.).

\section{Assessment of TNFxxx-a, IL-6, VEGF and CRP by ELISA}

TNF- $\alpha$, IL- 6 in liver tissues and serum VEGF, CRP levels were assessed quantitatively using enzyme linked immunosorbent assay (ELISA) kits as per the maker's guidelines.

\section{Assessment of oxidative stress parameters}

Liver of all the sacrificed animals were detached and kept aside on an autoclaved petridis along with ice cube. Liver tissues were converted into thin slices in chilled sucrose $(0.25 \mathrm{M})$ with the help of surgical scalpel and followed by quick blotting on a filter paper. Homogenization of minced tissues were achieved with $10 \mathrm{mM}$ Tris- $\mathrm{HCl}$ buffer, $\mathrm{pH} 7.4(10 \% \mathrm{w} / \mathrm{v})$ on a glass homogenizer followed by centrifugation at $0^{\circ} \mathrm{C}$ and speed was fixed at 10,000 X g. Resulting supernatant was assayed for endogenous antioxidant enzymes such as $\mathrm{GSH},{ }^{11}$ catalase $(\mathrm{CAT}),{ }^{12}$ superoxide dismutase $(\mathrm{SOD}){ }^{13}$ and malondialdehyde (MDA). ${ }^{14}$

\section{Histological study}

Liver tissues of sacrificed animals of aforementioned groups were detached and followed by cleaning with saline solution and stored in formalin (10\% phosphate buffer). 
Samples embedded on paraffin were sliced down into 5 $\mu \mathrm{m}$-thick segments and stained by means of hematoxylin and eosin (H\&E). Expected histopathological changes were assessed using light microscope (Olympus BX10, Tokyo, Japan) and photomicrographs were captured (Olympus DP12 camera, Japan). Information regarding treated animal group was not disclosed to pathologist executing histopathological assessment to acquire unbiased outcome.

\section{Statistical analysis}

All the statistical outcomes were stated in terms of mean \pm SEM. One-way ANOVA was performed and subsequently Bonferroni multiple comparison test was applied with the help of Prism, GraphPad version 5, GraphPad Software Inc., to evaluate statistical significance between more than two groups, where significance threshold was fixed at $p<.05$ for all assessment.

\section{RESULTS}

\section{Effect of SL or O3FAs or SL + O3FAs on ALT, AST and ALP}

Intraperitoneal administration of PCM triggered a noticeable fall in liver function portrayed by substantial $(p<0.001)$ elevation in ALT, AST and ALP levels in PCM treated rats when compared with normal control. SL or O3FAs or SL + O3FAs treated with PCM for 14 days period exhibited substantial $(p<0.001)$ reduction in ALT, AST and ALP values when compared with PCM treated rats. In Contrast, combination of SL and O3FAs exhibited marked $(p<0.001)$ alteration in ALT levels when compared with monotherapy with SL or O3FAs. However, co-administration of SL and O3FAs did not show a noticeable change in AST and ALP levels than monotherapy (Figure $1 \mathrm{~A}-\mathrm{C}$ ).

\section{Effect of SL or O3FAs or SL + O3FAs on total bilirubin, direct bilirubin and total protein}

PCM administration triggered a substantial $(p<0.001)$ elevation in total bilirubin, direct bilirubin and a substantial $(p<0.01)$ reduction in total protein when compared with normal control. Treatment with SL or O3FAs or SL + O3FAs exhibited substantial $(p<$ $0.001)$ reduction in total bilirubin, direct bilirubin levels when compared with PCM treated rats. In contrast, administration of SL or O3FAs did not show marked alteration in total protein levels when compared with PCM treated rats. Moreover, co-administration of SL and O3FAs did not show a noticeable change in
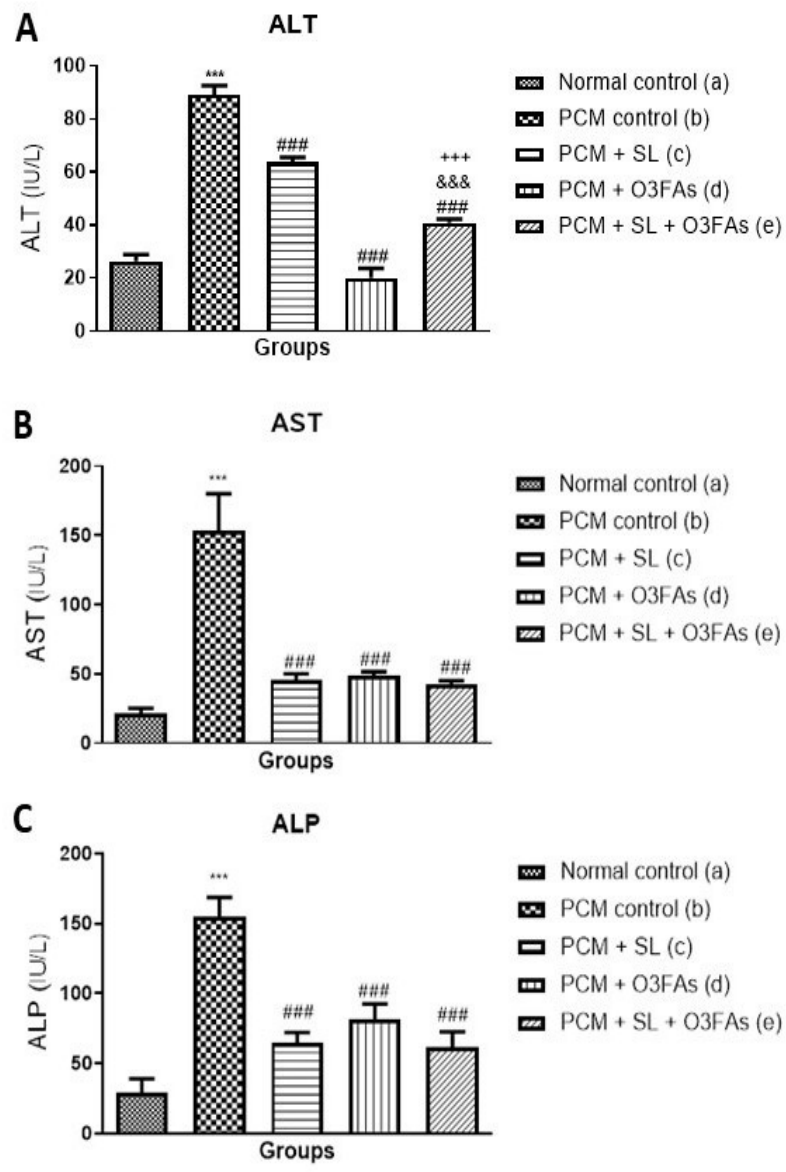

Figure 1: Effect of SL or O3FAs or SL + O3FAs on (A) ALT, (B) AST and (C) ALP.

All the statistical outcomes were stated in terms of mean \pm SEM; $n=6$ a vs. b, ${ }^{* * *}$ p $<0.001$; b vs. c, b vs. d and b vs. e, \#\#\# p < 0.001; c vs. e, \&\&\& p < $0.001 ;$ d vs.,+++ p $<0.001$

total bilirubin, direct bilirubin and total protein than monotherapy (Figure $2 \mathrm{~A}-\mathrm{C}$ ).

\section{Effect of SL or O3FAs or SL + O3FAs on oxidative stress biomarkers in kidney tissue}

A substantial $(p<0.001)$ rise in MDA content and reduced activity of SOD $(p<0.001)$, CAT $(p<0.001)$ and GSH $(p<0.01)$ levels were noticed after providing PCM treatment. This indicates the production of free radicals and role of oxidative stress in liver toxicity induced as a result of PCM treatment.

It was observed a substantial $(p<0.05 ; p<0.001$; $p$ $<0.001)$ reduction in MDA content and an elevation in the level of antioxidant enzymes, SOD $(p<0.001$; $p<0.001 ; p<0.001)$, CAT $(p<0.001 ; p<0.001 ; p<$ $0.001)$ and GSH $(p<0.01 ; p<0.01 ; p<0.01)$ levels after providing treatment with SL or O3FAs or SL + O3FAs, respectively. Whereas it was also showed that treatment of SL with O3FAs showed more substantial $(p<0.05)$ decline in MDA levels as compare to rats treated with SL alone. However, combination therapy of SL along with 

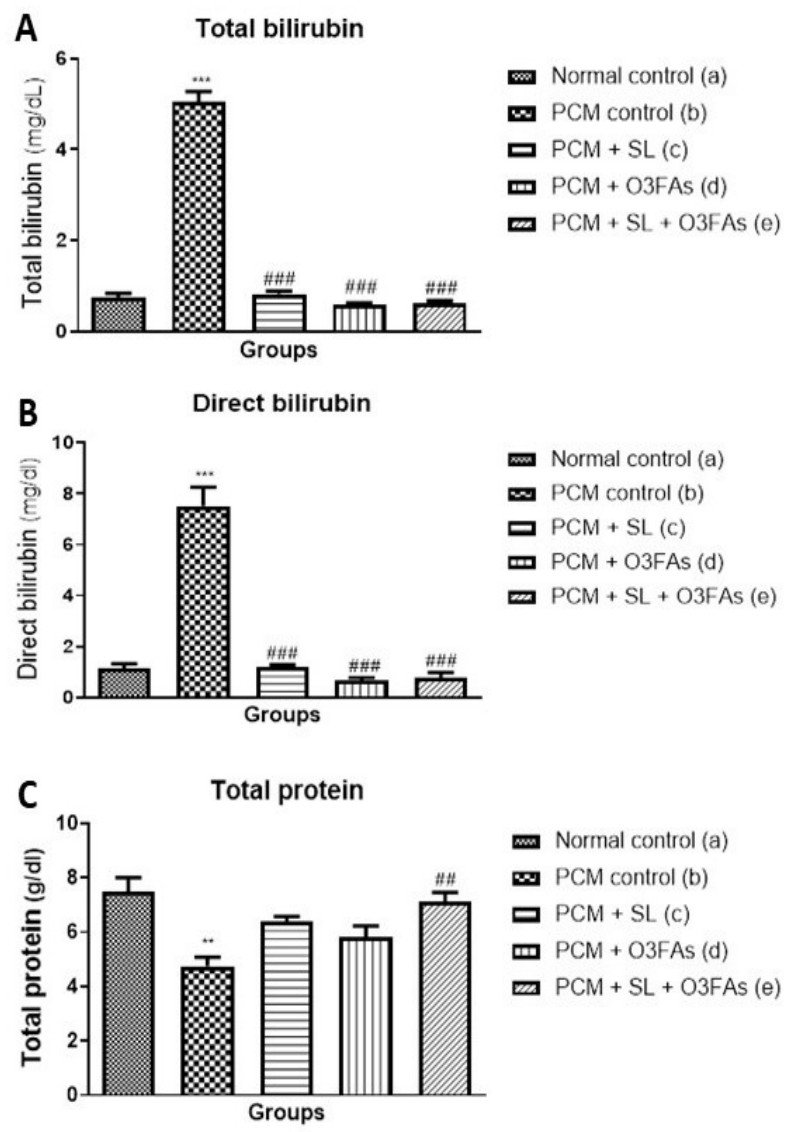

Figure 2: Effect of SL or O3FAs or SL + O3FAs on (A) Total bilirubin, (B) Direct bilirubin and (C) Total protein.

All the statistical outcomes were stated in terms of mean \pm SEM; $n=6$ a vs. b, ${ }^{*} p<0.01,{ }^{* * *} p<0.001$; b vs. c, b vs. d and b vs. e, $\# p<0.05, \# \# p<0.01$, $\# \# \# p<0.001$
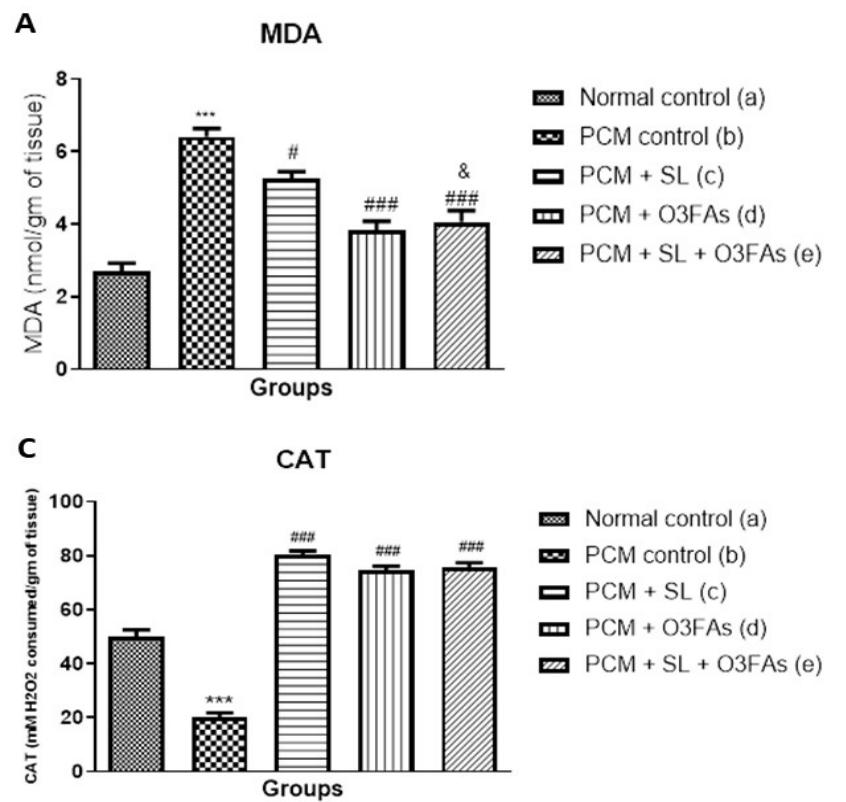

O3FAs showed substantial alterations in SOD levels when O3FAs given exclusively (Figure 3 A-D).

\section{Effect of SL or O3FAs or SL + O3FAs on TNF-a, IL- 6, VEGF and CRP}

Administration of PCM exhibited substantial rise in renal TNF- $\alpha(p<0.01)$, IL-6 $(p<0.001)$ and serum VEGF $(p<0.001)$ and CRP $(p<0.001)$ content. Administration of SL or O3FAs or SL + O3FAs with PCM for 14 days period substantially reduced TNF- $\alpha$ $(p<0.001 ; p<0.001 ; p<0.001)$, IL-6 $(p<0.001 ; p$ $<0.001 ; p<0.001)$, VEGF $(p<0.05 ; p<0.05 ; p<$ $0.01)$ and $\mathrm{CRP}(p<0.001 ; p<0.01 ; \mathrm{p}<0.001)$ when compared with PCM treated rats, respectively. Though, concomitant administration of SL with O3FAs did not have any considerable alteration in TNF- $\alpha$, IL-6, VEGF and CRP contents when compared with monotherapy of SL or O3FAs (Figure 4 A-D).

\section{Histological studies}

In the proposed research protocol, the histological variations in liver in all groups were assessed. Liver segments of the normal rats exhibited regular orientation of liver, whereas PCM control rats showed fatty changes and dilation of central vein. Treatment with SL or O3FAs and combination of both showed no gross anatomical changes were found (Figure 5 A-E).

\section{DISCUSSION}

The protective effects of SL or O3FAs or SL + O3FAs against hepatotoxicity initiated by PCM were investigated

B

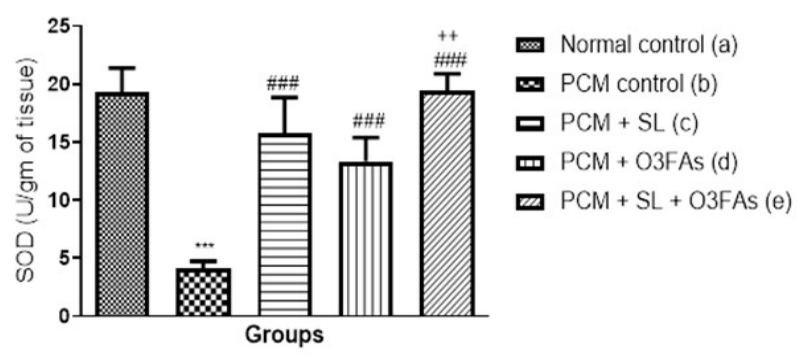

D

GSH

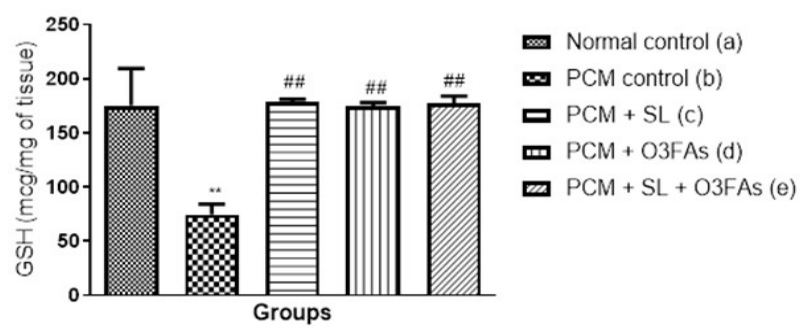

Figure 3: Effect of SL or O3FAs or SL + O3FAs on (A) MDA, (B) SOD, (C) CAT and (D) GSH.

All the statistical outcomes were stated in terms of mean \pm SEM; $n=6$

a vs. b, ${ }^{* *} P<0.01,{ }^{* * *} P<0.001$; b vs. c, b vs. $\mathrm{d}$ and b vs. e, $\# p<0.05, \# \# p<0.01$, \#\#\# $p<0.001$; c vs. e, \& $p<0.05$; d vs. e, $++p<0.01$ 
A

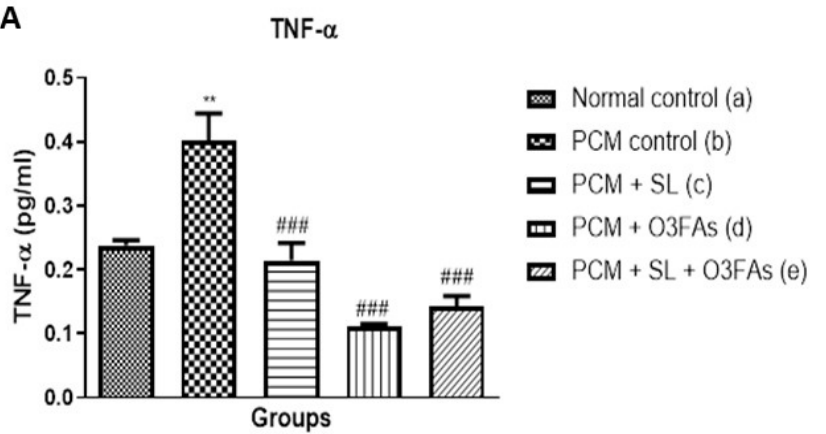

C

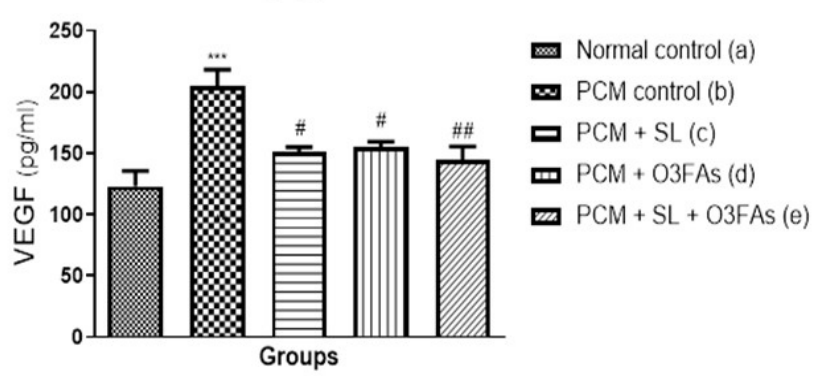

B

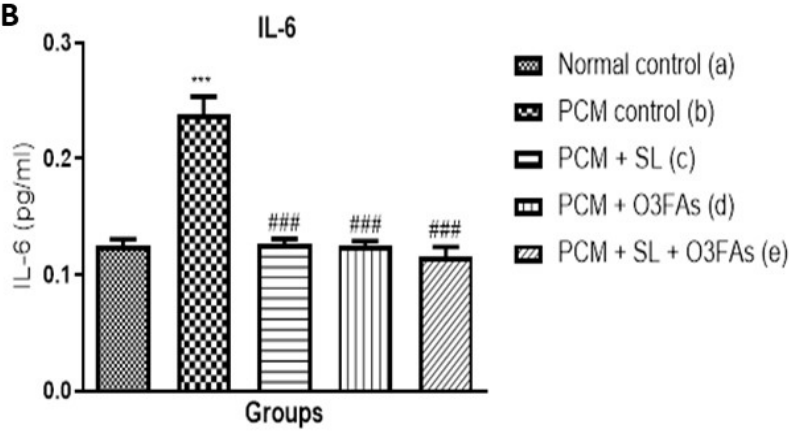

D CRP

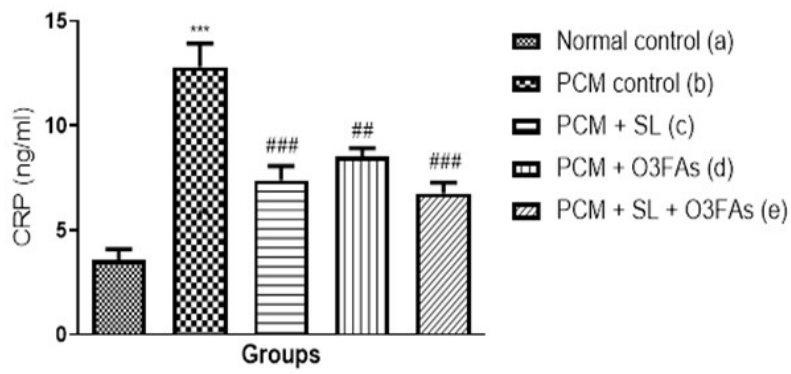

Figure 4: Effect of SL or O3FAs or SL + O3FAs on (A) TNF-a, (B) IL-6 (C) VEGF and (D) CRP.

All the statistical outcomes were stated in terms of mean \pm SEM; $n=6$

a vs. b, ${ }^{* *} \mathrm{P}<0.01,{ }^{* * *} \mathrm{P}<0.001$; b vs. c, b vs. d and b vs. e, \#p $<0.05, \# \# \mathrm{p}<0.01, \# \# \# \mathrm{p}<0.001$.

in the present study. Our reports showed that a single intraperitoneal injection of PCM caused liver injury in rats, exhibited by a substantial raise in serum AST, ALT, ALP, total bilirubin and direct bilirubin levels as well as the histopathological changes. Higher levels of abovementioned biomarkers result in revealing of cellular escape and loss of function of liver cellular membrane. These elevated levels of biomarkers are reliable with the conclusions of numerous earlier studies. Histopathological changes with necrosis, degenerated hepatocytes, inflammatory cells infiltration and dilated hepatic sinusoids are evident in PCM-intoxicated rats. ${ }^{15,16}$ The current results prove that treatment of monotherapy and combination therapy of SL or O3FAs potentially attenuated PCM-initiated liver injury. These outputs are accordance with earlier reports that stated that $\mathrm{SL}^{17}$ and O3FAs ${ }^{18}$ lessened serum ALP, ALT, AST, total bilirubin and direct bilirubin levels and histopathological changes in rats.

In the current work, substantial rise was observed in MDA content and reduced action of SOD, GSH and CAT after providing PCM treatment. This indicates the production of free radicals and role of oxidative stress in liver toxicity induced as a result of PCM treatment. These results are in accordance with the previous studies which reported that elevation in lipid peroxidation and decrease of glutathione content, SOD activity and

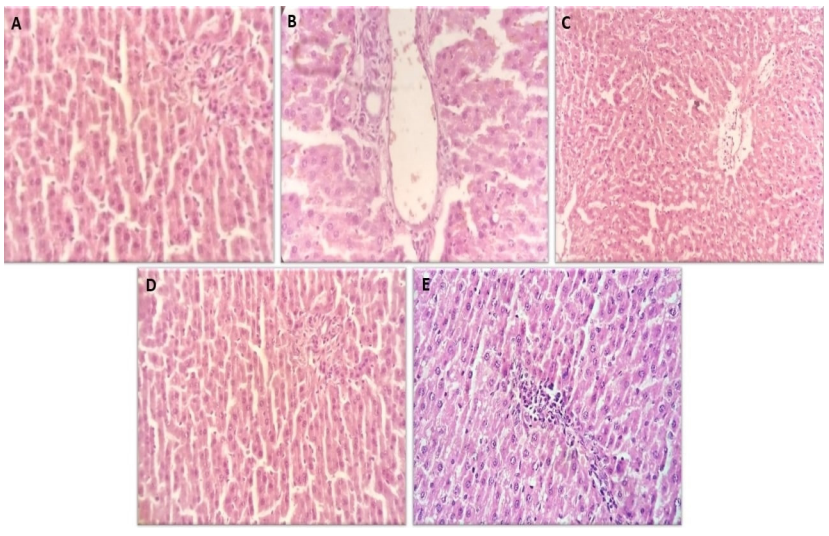

Figure 5: Effect of SL or O3FAs or SL + O3FAs on histopathology of liver tissue (A) Normal Control, (B) PCM Control, (C) PCM + SL (D) PCM + O3FAs and (E) PCM + SL + O3FAs.

catalase activity is an indicator of oxidative stress which is responsible for liver injury. ${ }^{19-21}$

It was observed a substantial decline in MDA level and raise of antioxidant enzymes (SOD, GSH and CAT) were experienced after the administration of SL or O3FAs. Significant reduction in generation of free radicals after treatment with SL or O3FAs in liver toxicity indicates the protective role of them against oxidative stress. These results are further confirmed by earlier the study in which SL was able to decrease MDA and raised the levels of SOD, CAT and GSH in $\mathrm{CCl}_{4}$ hepatotoxicity 
induced rats. ${ }^{18,22,23}$ In contrast, combination therapy of SL along with O3FAs showed remarkable alterations in SOD or MDA content than individual therapy of O3FAs or SL, respectively.

It was previously demonstrated that during hepatotoxicity, the harmed cells liberates a group of cytokines which is also responsible for the discharge additional inflammatory mediators from Kupffer cells. ${ }^{24}$ In our report, administration of PCM exhibited a substantial rise in the liver TNF- $\alpha$, IL- 6 and serum CRP levels, demonstrating inflammation. These inflammatory reactions are generally seemed by biliary obstruction ${ }^{25}$ and are associated in raised indicators of end-organ damage and death. TNF- $\alpha$, IL- 6 and CRP are an inflammatory mediator contributes to the pathological complications observed in several diseases. ${ }^{26}$ Administration of SL or O3FAs in PCM treated rats for 14 days meaningfully reduced liver TNF- $\alpha$, IL- 6 and serum CRP levels. These findings are in a line with previous study which shows that supplementation of omega-3 FAs in HIV-infected patients significantly decreases serum CRP levels when compared to the control group indicating the use of O3FAs in the treatment of inflammatory disorders.$^{27-}$ ${ }^{29}$ In contrast, combination therapy of SL along with O3FAs did not show remarkable alterations in liver TNF- $\alpha$, IL-6 and serum CRP levels than individual therapy.

Elevation in expression of VEGF and its receptors has been stated in experimentally-initiated cirrhosis ${ }^{30}$ and $\mathrm{CCl}_{4}$-initiated fibrosis. ${ }^{31}$ Also, it was reported that VEGF is playing a major part in the development of liver fibrosis through making the proliferation of sinusoidal endothelial cells and Hepatic Stellate Cells (HSCs). ${ }^{32}$ In our study, we found that serum levels of VEGF were diminished when PCM-initiated rats treated with SL or O3FAs or SL + O3FAs. Therefore, it appears that decreased production of VEGF by SL or O3FAs or $\mathrm{SL}+\mathrm{O} 3 \mathrm{FAs}$ plays a vital role in reducing collagen production in HSCs.

\section{CONCLUSION}

Our study concluded that SL and/or O3FAs protected the liver against PCM-initiated liver toxicity via attenuating inflammation, oxidative stress, cytokines induced damage. However, the substantially increased hepatoprotective effects of SL when co-administration with O3FAs indicate a possible synergism.

\section{ACKNOWLEDGEMENT}

We are sincerely thankful to Sumandeep Vidyapeeth (Deemed to be University) for providing financial support to carry out the study.

\section{CONFLICT OF INTEREST}

The authors declare that there is no conflict of interest.

\section{ABBREVIATIONS}

SL: Silymarin; PCM: Paracetamol; O3FAs: Omega-3 fatty acids; ROS: Reactive Oxygen Species; IL 6: Interleukin 6; TNF- $\alpha$ : Tumor Necrosis Factor-alpha; VEGF: Vascular endothelial growth factor; CRP: C - reactive protein; ALP: Alkaline phosphatase; ALT: Alanine transaminase; AST: Aspartate transaminase; IAEC: Institutional Animal Ethics Committee; CPCSEA: Committee for the purpose of Control and Supervision of Experiments on Animals; ELISA: Enzyme Linked Immunosorbent Assay; MDA: Malondialdehyde; SOD: Superoxide Dismutase; CAT: Catalase; GSH: Glutathione; SEM: Standard Error of the Mean.

\section{REFERENCES}

1. Rumack BH. Acetaminophen misconceptions. Hepatology. 2004;40(1):10-5.

2. Prescott LF. Hepatotoxicity of mild analgesics. $\mathrm{Br} \mathrm{J} \mathrm{Clin} \mathrm{Pharmacol.}$ 1980;10(S2):373S-9S.

3. Jaeschke $H$, McGill MR, Ramachandran A. Oxidant stress, mitochondria and cell death mechanisms in drug-induced liver injury: Lessons learned from acetaminophen hepatotoxicity. Drug Metab Rev. 2012;44(1):88-106.

4. Knight TR, Ho YS, Farhood A, Jaeschke H. Peroxynitrite is a critical mediator of acetaminophen hepatotoxicity in murine livers: Protection by glutathione. $J$ Pharmacol Exp Ther. 2002;303(2):468-75.

5. Shahani S. Evaluation of hepatoprotective efficacy of APCL-A polyherbal formulation in vivo in rats. Indian Drugs. 1996;36:628-31.

6. Venkateswaran S, Pari L, Viswanathan P, Menon VP. Protective effect of Livex, a herbal formulation against erythromycin estolate-induced hepatotoxicity in rats. J Ethnopharmacol. 1997;57(3):161-7.

7. Wagner H, Diesel P, Seitz M. The chemistry and analysis of silymarin from Silybum marianum Gaertn. Arzneimittelforschung 1974;24(4):466-71.

8. Hau DK, Wong RS, Cheng GY, Wong WY, Tong SW, Chan KW, et al. Novel use of silymarin as delayed therapy for acetaminophen-induced acute hepatic injury. Complement Med Res. 2010;17(4):209-13.

9. Meganathan M, Gopal KM, Sasikala P, Mohan J, Gowdhaman N, Balamurugan K, Nirmala P, et al. Evaluation of hepatoprotective effect of omega 3-fatty acid against paracetamol induced liver injury in albino rats. Global J Pharmacol 2011;5(1):50-3.

10. Senthilkumar R, Chandran R, Parimelazhagan T. Hepatoprotective effect of Rhodiola imbricata rhizome against paracetamol-induced liver toxicity in rats. Saudi J Biol Sci 2014;21(5):409-16.

11. Moron MS, Depierre JW, Mannervik B. Levels of glutathione, glutathione reductase and glutathione S-transferase activities in rat lung and liver. Biochim Biophys Acta. 1979;582(1):67-78.

12. Aebi H. Catalase in vitro. Methods Enzymol. 1984;105:121-6. 
13. Misra HP, Fridovich I. The role of superoxide anion in the autoxidation of epinephrine and a simple assay for superoxide dismutase. J Biol Chem. 1972;247(10):3170-5.

14. Slater TF, Sawyer BC. The stimulatory effects of carbon tetrachloride and other halogenoalkanes on peroxidative reactions in rat liver fractions in vitro. General features of the systems used. Biochem J 1971;123(5):805-14.

15. Mishra G, Khosa RL, Singh P, Jha KK. Hepatoprotective potential of ethanolic extract of Pandanus odoratissimus root against paracetamol-induced hepatotoxicity in rats. J Pharm Bioallied Sci. 2015;7(1):45-8.

16. EIFaras AA, Elsawaf AL. Hepatoprotective activity of quercetin against paracetamol-induced liver toxicity in rats. Tanta Med J. 2017;45(2):92-8.

17. Freitag AF, Cardia GF, DaRocha BA, Aguiar RP, Silva-Comar FM, Spironello $\mathrm{RA}$, et al. Hepatoprotective effect of silymarin (Silybum marianum) on hepatotoxicity induced by acetaminophen in spontaneously hypertensive rats. Evid Based Complementary Altern Med. 2015. Article ID 538317.

18. Adeyemi WJ, Olayaki LA. Diclofenac-induced hepatotoxicity: Low dose of omega-3 fatty acids have more protective effects. Toxicol Rep. 2018;5:90-5.

19. Kamiyama T, Sato C, Liu J. Role of lipidperoxidation in acetaminophen induced hepatotoxicity: Comparison with carbontetrachloride. Toxicol Lett. 1993;66(1):7-12.

20. Recknagel RO. Carbon tetrachloride hepatotoxicity. Pharmacol Rev. 1967;19(2):145-208

21. Brattim WJ, JrGlenda EA, Recknagel RO. Pathological mechanism in carbon tetrachloride hepatotoxicity. J Free Radicals Biol Med. 1985;1(1):27-38.

22. Maheshwari R, Pandya B, Balaraman R, Seth AK, Yadav YC, Sankar VS. Hepatoprotective effect of Livplus-A polyherbal formulation. Pharmacogn J. 2015;7(5):311-6.

23. Pradeep K, Mohan CVR, Gobianand K, Karthikeyan S. Silymarin: An effective hepatoprotective agent against diethylnitrosamine-induced hepatotoxicity in rats. Pharm Biol. 2007;45(9):707-14.
24. Luckey SW, Petersen DR. Activation of Kupffer cells during the course of carbon tetrachloride-induced liver injury and fibrosis in rats. Exp Mol Pathol. 2001;71(3):226-40.

25. Minter RM, Bi X, Ben-Josef G, Wang T, Hu B, Arbabi S, et al. LPS-binding protein mediates LPS-induced liver injury and mortality in the setting of biliary obstruction. Am J Physiol Gastrointest Liver Physiol. 2009;296(1):G45-54.

26. Feng R, Lu Y, Bowman LL, Qian Y, Castranova V, Ding M. Inhibition of activator protein-1, NF-kappaB and MAPKs and induction of phase 2 detoxifying enzyme activity by chlorogenic acid. J Biol Chem. 2005;280(30):27888-95.

27. Shalan MG, Mostafa MS, Hassouna MM, El-Nabi SH, El-Refaie A. Amelioration of lead toxicity on rat liver with Vitamin $\mathrm{C}$ and silymarin supplements. Toxicol 2005;206(1):1-5.

28. Shaker E, Mahmoud H, Mnaa S. Silymarin, the antioxidant component and Silybum marianum extracts prevent liver damage. Food Chem Toxicol. 2010;48(3):803-6.

29. Morvaridzadeh $M$, Sepidarkish $M$, Yavari M, Tahvilian N, Heydarian A, Khazdouz $M$, et al. The effects of omega-3 fatty acid supplementation on inflammatory factors in HIV-infected patients: A systematic review and metaanalysis of randomized clinical trials. Cytokine. 2020;136:155298

30. Corpechot C, Barbu V, Wendum D, Kinnman N, Rey C, Poupon R, et al. Hypoxia-induced VEGF and collagen I expressions are associated with angiogenesis and fibrogenesis in experimental cirrhosis. Hepatology. 2002;35(5):1010-21.

31. Shi H, Dong L, Bai Y, Zhao J, Zhang Y, Zhang L. Chlorogenic acid against carbon tetrachloride-induced liver fibrosis in rats. Eur $\mathrm{J}$ Pharmacol. 2009;623(1-3):119-24.

32. Yoshiji H, Kuriyama S, Yoshii J, Ikenaka Y, Noguchi R, Hicklin DJ, et al. Vascular endothelial growth factor and receptor interaction is a prerequisite for murine hepatic fibrogenesis. Gut. 2003;52(9):1347-54.

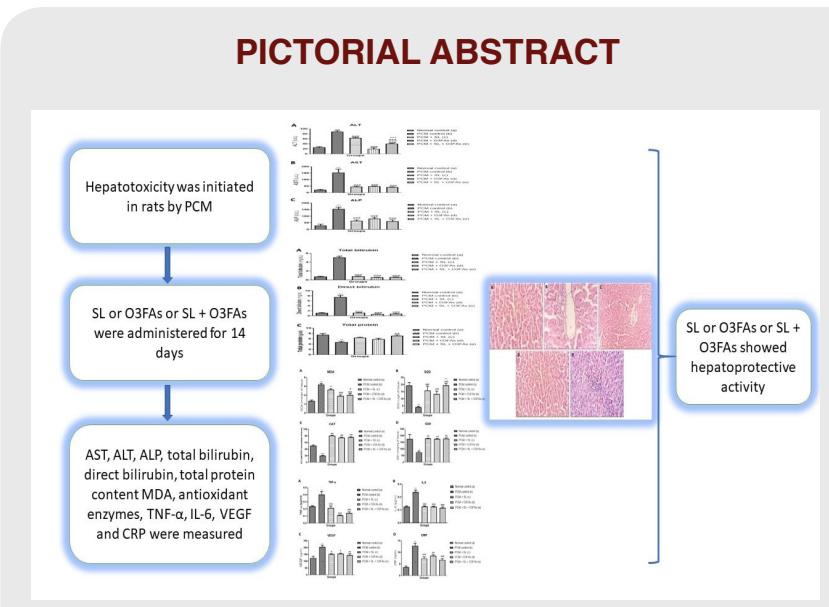

\section{SUMMARY}

The current study demonstrated the hepatoprotective role of SL and O3FAs against PCM-initiated liver toxicity in wistar rats. In this study, SL and O3FAs substantively ameliorates PCM-initiated liver toxicity mainly by decreasing the levels of TNF- $\alpha$, IL-6, CRP, VEGF along with suppressing the increased liver markers and potentiate the depleted antioxidant enzymes caused by PCM.

\section{About Authors}

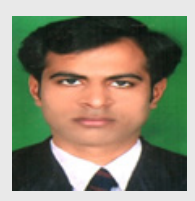

Dr. Rajesh A. Maheshwari, is Professor in Department of Pharmacy, Sumandeep Vidyapeeth (Deemed to be University), Vadodara, India. He has also been honored with Member of National Academy of Medical Sciences, India (MAMS) in 2017 for his contribution towards the Medical Research. In addition to this, he was also been conferred for the "Sumandeep Vidyapeeth Research Award" for two times i.e. in 2015-16 and 2018-19. 


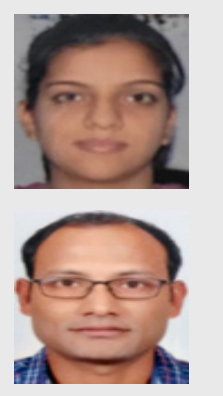

Ms. Divya Khemani, was a post graduate student, completed her PG in the division of Pharmacology, Department of Pharmacy, Sumandeep Vidyapeeth (Deemed to be University), Vadodara, India.

Dr. Ashim Kumar Sen, currently working as Professor in Department of Pharmacy, Sumandeep Vidyapeeth (Deemed to be University), Vadodara, India.

Dr. R. Balaraman, FAMS is one of the senior and eminent pharmacologists. He has over 44 years of experience in teaching and research. He is currently working as the Emeritus Professor in the Dept of Pharmacy, Sumandeep Vidyapeeth, Piparira, Vadodara. In 2002 he was elected as the Member of National Academy of Medical Science (MAMS) He was subsequently elected as a Fellow of The Academy ofMedical Science (FAMS) for the year 2009. He received several awards like Dr. Uvanas prize, P. P. Suryakumari prize and O. D. Gulati prize for his contribution in cardiovascular research. He has served as a Vice-President of The Indian Pharmacological Society (India) in 2008. He was the Executive committee member of 4th World Congress of IACS held in 2011.

Dr. Ramaswamy Velmurugan, Currently working as Senior Scientist in Century Pharmaceuticals Limited, Vadodara, India. He has published more than 10 research papers in reputed journals.

Dr. Dhanya B. Sen, Currently working as Associate Professor in Department of Pharmacy, Sumandeep Vidyapeeth (Deemed to be University), Vadodara, India.

Cite this article: Maheshwari RA, Khemani D, Sen AK, Balaraman R, Velmurugan R, Sen DB. Protective Effects of Silymarin and/or Omega-3 Fatty Acids against Paracetamol-initiated Liver Toxicity in Rats. Indian J of Pharmaceutical Education and Research. 2022;56(1s):s81-s88. 